\title{
Evaluation of the Use of Inertial Platform for the
}

\section{Generation of Cartography}

\author{
Luís Antônio de Lima ${ }^{1}$, Diogenes Cortijo Costa $^{2}$, Jorge Luiz Alves Trabanco ${ }^{2}$ and Maria Teresa Françoso ${ }^{2}$ \\ 1. Technical Department, Fototerra Atividades de Aerolevantamento Ltda (Fototerra Aerial Survey Activities Ltd.), San Paulo \\ 03337-000, Brazil \\ 2. Department of Geotechnics and Transportation, The State University of Campinas, San Paulo 13083-070, Brazil
}

\begin{abstract}
This study aims to analyze the quality of positioning orthophoto generated through the technique of direct georeferencing using metric digital camera system coupled to the laser system, and both systems were aided inertial navigation platform. For this, we compared the coordinates obtained in 16 control points collected in the field with dual-frequency GNSS (global navigation satellite system) receiver with those obtained in the orthophoto, flight conducted in June 2009 on the campus of the State University of Campinas-UNICAMP, using a medium format digital camera and laser system, with which we obtained images with a spatial resolution of $0.15 \mathrm{~m}$. Taking into account the pattern accuracy cartographic used in Brazil, it is concluded that the products produced have a standard accuracy "A" to 1:2,000 scale, which represents the best quality level, both for planimetric as for altimetry, and that the procedure reached results consistent with cartographic products in 1:2,000 scale, representing a reduction of steps in the mapping process, necessary for the preparation of cartographic databases with reduction of time for preparation of design basis large civil engineering projects, such as roads, railways, studies of urban road systems, power plants and other infrastructure systems needed to develop the country.
\end{abstract}

Key words: Orthophotos, photogrammetry, GNSS.

\section{Introduction}

Since the 1990s, little has been invested towards improving cartographic maps in Brazil. However, advances in digital cartography require state or private initiatives to resume map generation.

A large portion of the information we need is geographical in nature (position), and maps and cartography are the means of communicating this information and, therefore, must be better used. Innumerable reasons lead many users to abandon cartographic information, the primary being the large limitations on map production. The speed of automated digital mapping provides innumerable advantages for urban planning activities because planners finally see maps and cartographic images as easily assimilated graphical media for representing

Corresponding author: Luís Antônio de Lima, M.Sc., civil engineer, research fields: geodesy, surveying and photogrammetry. E-mail: luis@fototerra.com.br. and analyzing spatial information. Automation techniques allow situations to be diagnosed and simulated before becoming obsolete and outdated [1].

Potential users, such as the public administration, are discouraged from using maps and cartography due to delays and high production costs, the few maps and cartographic records that do exist date from the 1970s and 1980s and are entirely outdated, unreliable and on an inappropriate scale.

Currently in Brazil, there are several companies working in the area of mapping, the vast majority using digital equipment, however, the process of systematic use of inertial platform is not widespread among companies nor in some of the universities.

The growing demand for infrastructure, which is characterized by large civil engineering works such as highways, railways, dams and urban development plans, and the implementation of road systems, which demand the opening of structural paths, bridges and 
viaducts, require reductions in the cost and time needed to expand digital cartographic databases, which are essential inputs for designing these large works.

Currently, basic cartography occurs via the classical methods of topographical and geodesic surveying, using equipments such as theodolites, total stations, levels and the GNSS (global navigation satellite system). Other methods for developing cartographic databases include satellite images or aerial surveys using passive or active sensors, each of which has advantages and disadvantages. The process of generating cartographic databases using aerial survey processes with sensors such as aerial photogrammetric cameras (analog or digital), laser systems (light detection and ranging), aerially transported radars or satellite imaging can be divided into four stages: image acquisition (via either analog or digital methods), field support for georeferencing data positioning, image orthorectification or geometric correction and cartographic database generation via restitution.

The geometric correction process normally referred as image georectification, traditionally over the last few years, the concept of image georeferencing has been based on aerial triangulation, which needs the presence of GCP (ground control points) in the project area $[2,3]$.

This study evaluates the use and accuracy of an inertial navigation system, which involves the image acquisition stage of the aerial survey process, with the objective of considerably decreasing the field support, positioning and orthorectification stages to make the cartographic generation process more effective and reduce both the time requirements and cost. The inertial navigation systems can be used with both digital and analog technologies. The purpose of this system is to obtain positioning data and information necessary to execute external orientation immediately after capturing the image/information. In other words, Cartesian coordinates and attitude of the photogrammetric system ( $\omega$-omega, $\varphi-$ phi and $\kappa$-kappa) combined with the inertial is necessary to use either the GNSS or airplane data. The GNSS is responsible for measuring Cartesian coordinates while capturing the images [4].

This study was evaluated via on-site verification (field) of the flight performed in June 2009 over the campus of the State University of Campinas (Universidade Estadual de Campinas-Unicamp) using a medium-format aerial digital camera and laser scanner system to obtain images with a $0.15 \mathrm{~m}$ spatial resolution and a digital model of the ground with a $0.50 \mathrm{~m}$ resolution. In the analyzed area, 16 photo-identifiable control points were selected and measured in the field using dual-frequency L1/L2 GNSS receivers.

\section{Direct Georeferencing Process}

Most projects using aerial photos require executing the geometric correction and positioning of the photo/image to extract the measurements and coordinates of a given projection system.

Therefore, the IO (interior orientation) and EO (exterior orientation) parameters must be obtained.

According to Brito and Coelho [5], "the interior orientation is the reconstruction of the perspective beam, which is, referencing the image relative to the camera. Once the images obtained are isolated from the others and saved as digital files without any metric information (that is, only using the pixel coordinate system of the digital images themselves), the internal camera-image system corresponding to the moment of the photographs can be reconstituted. Only in this manner will the photographic images be measured with precision".

The interior orientation only consists of placing images together in positions similar to those of the camera at the moment they were obtained.

For digital photogrammetry, the interior orientation parameters are determined via software with the final system (photo-camera) being expressed by the 
photographic coordinates of the fiducial marks and calibrated focal distance, which certifies the camera calibration, adjusted to determine the transformation coefficients between the two systems. Thus, any point which belonged to the image space can be positioned in a Cartesian coordinate system [5].

The EO parameters were connected to the camera position and orientation at the instant the images/photos were taken and are obtained in either a direct or indirect manner using the control points in the field. The objective is to determine the position and attitude of each photo relative to the reference in object space according to Fig. 1.

According to Brito and Coelho [5], an image is externally oriented if six external orientation parameters are known: the sensor's object space coordinates of perspective and the angles of rotation and attitude, $\omega, \varphi$ and $\kappa$ (omega, phi and kappa), as shown in Fig. 2, where, $X$ corresponds to the center of perspective in the $X$ axis of object space; $Y$ corresponds to the center of perspective in the $Y$ axis of object space; $Z$ corresponds to the center of perspective in the $Z$ axis of object space.

The Euler angles, $\omega, \varphi$ and $\kappa$ (omega, phi and kappa), represent the rotations of the local coordinate system, $x, y$ and $z$, relative to the global system for the ground, $X, Y$ and $Z$. Rotating $x, y$, and $z$, by $-\omega,-\varphi,-\kappa$, makes it parallel to $X, Y$ and $Z$ [5]:

$\Omega$ represents the rotation of the $x$ axis relative to $X$;

$\Phi$ represents the rotation of the $y$ axis relative to $Y$;

$K$ represents the rotation of the $z$ axis relative to $Z$.

In this study, the EO parameters were determined in real time or post-processed, using satellite positioning systems and an inertial measurement unit; this approach is known as direct georeferencing.

The direct georeferencing of images has some advantages relative to traditional orientation processes, including the automation of the rectification and positioning stages, ease of developing cartographic databases at locations where field support is restricted and the improved control of aerial shots during flight.
According to Cramer [6], the technique of direct georeferencing without the use of any control point has great results, but you must determine a number of checkpoints for the implementation of quality control of the final product, a process carried out in this article.

\section{Image Capture Process and GNSS/IMU Data}

To analyze the inertial navigation system as a resource for direct georeferencing, as previously cited, a photogrammetric flight authorized by the Ministry of Defense (authorization number 106/2009) and AVOEM - permit to fly the largest state of aeronautics (number 678/09 given by the staff of the Armed Forces (EMFA-Estado Maior das Forças Armadas)) was conducted. The following equipment was used: two medium-format Aplannix DSS 32 digital aerial photogrammetric cameras (one for capturing RGB — red, green and blue —images and

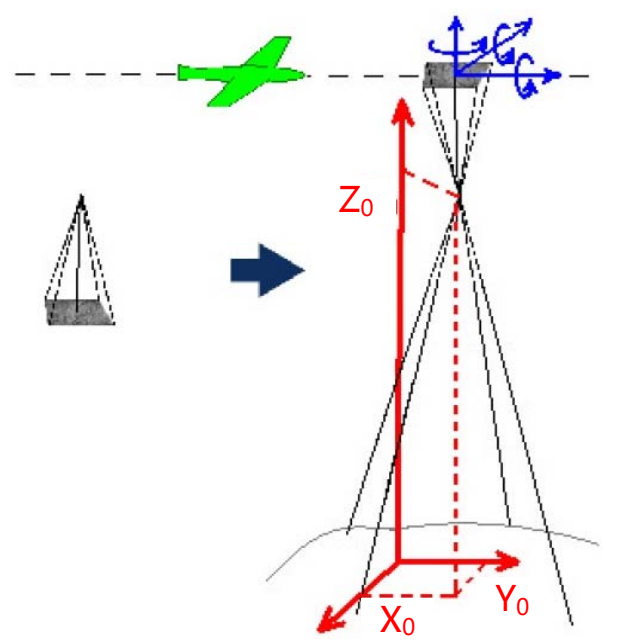

Fig. 1 Objective exterior orientation [5].

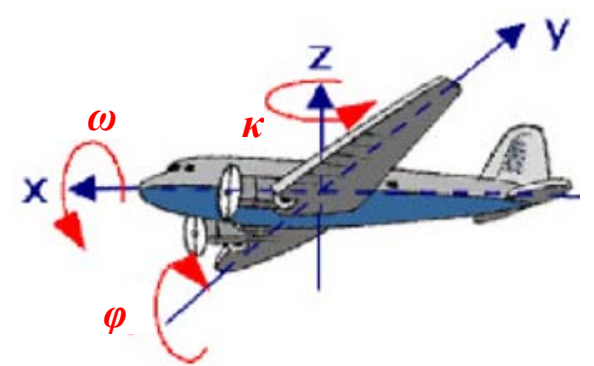

Fig. 2 Attitude angles of the airplane [5]. 
other for infrared images) with a focal distance of 60 $\mathrm{mm}$ and a resolution of 22 megapixels, an Aplannix 410 inertial navigation system, a Toposys Harrier 56 laser scanner system, a Piper Seneca airplane-EPN registration and two GNSS L1/L2 Novatel model DL4 plus receivers.

For reference, the mark labeled "M03", which pertains to the registration reference network (Rede de Referência Cadastral) at the State University of Campinas (Universidade Estadual de Campinas-UNICAMP) whose UTM (Universal Transverse Mercator) coordinates in the WGS (World Geodetic System) 84 datum are $N=7,475,414.8995$, $E=188,165.4207$ with an ellipsoidal height of 614,537. The flight in question occurred on July 9, 2009. To cover the entirety of the UNICAMP campus, seven passes were necessary with an average height of $550 \mathrm{~m}$ above ground and using an Aplannix DSS model medium-format camera with a resolution of $9.00 \mu \mathrm{m}$, a focal frame with width of $48.92 \mathrm{~mm}$ and length of $36.83 \mathrm{~mm}$, and a focal distance of $60 \mathrm{~mm}$. The approximate flight scale is $1: 9,150$ and the pixel size was $0.082 \mathrm{~m}$ at the moment of capture.

It is important to emphasize that during image capture, the aerial photogrammetric camera, GNSS within the airplane and the IMU (inertial measurement unit) were synchronized using a specific software program developed by Aplannix. This program emits and automatically records a signal the moment a photo is taken (opening of the camera shutter) to integrate the GNSS and camera attitude data of the inertial system.

\section{Data Processing}

After collecting the flight data, three distinct stages of processing yielded the final data.

The GNSS was differentially corrected using the data recorded by the internal airplane equipment and the data obtained using equipment at point M03 (UNICAMP Campus).

The GNSS data were combined with data from the inertial system, $\omega, \varphi$ and $\kappa$ (omega, phi and kappa). This processing stage uses the camera attitude and EO data for each instant captured in the GNSS system.

The photographic events were combined with the EO data.

Thus, a set of aerial photos can be connected to a table of the EO data as shown in Table 1.

The data processing was performed using the POS EO computation utility software (version 4.4) developed by Aplannix, which relates the in-flight photos to the EO data extracted from the GNSS and inertial systems.

For the studied case, a mesh of points from the laser scanner system and airplane was coupled to elaborate the digital ground model with a precision comparable to the cartography of interest. The details of this process are not addressed in this study, however, the accuracy of the final results was evaluated.

The orthophotos and final mosaic were processed using the aerial photos and EO parameters shown in Table 1, the digital ground model generated using the laser system and the interior orientation parameters extracted from the camera calibration certificate.

Thus, with the use of direct georeferencing process, there was a radical change in the orientation of the imaging process, which is oriented independently [7].

\section{Results and Discussions}

To analyze and evaluate the process used, 16 control points within the Unicamp campus in Campinas were selected to obtain the best possible geometry and guarantee their simple identification in the orthophotos, which allow the differences between coordinates captured in the field and measured in the orthophoto to be analyzed. The point distribution is shown in Fig. 3, that is the end product (orthophoto) and the distribution of the control points used to verify the quality of the final mapping.

The control points were surveyed using a Topcon GNSS receiver, HIPER LITE + model, with two frequencies via the differential method referencing 
Table 1 EO data.

\begin{tabular}{llllllll}
\hline Photo & Time & $X$ & $Y$ & $Z$ & Roll $\omega$ & Pitch $\varphi$ & Heading $\kappa$ \\
\hline 0545710 & $396,554.176$ & $3,992,798.04$ & $-4,315,777.74$ & $-2,467,078.262$ & 1.03508 & 2.82360 & 246.10231 \\
0545750 & $396,558.228$ & $3,992,626.91$ & $-4,315,877.18$ & $-2,467,197.362$ & 0.49988 & 2.45384 & 245.75876 \\
0545790 & $396,562.262$ & $3,992,455.98$ & $-4,315,974.22$ & $-2,467,314.060$ & 2.18213 & 1.87089 & 245.86012 \\
0545820 & $396,565.732$ & $3,992,308.54$ & $-4,316,058.20$ & $-2,467,412.988$ & -0.87679 & 1.58416 & 246.47499 \\
\hline
\end{tabular}

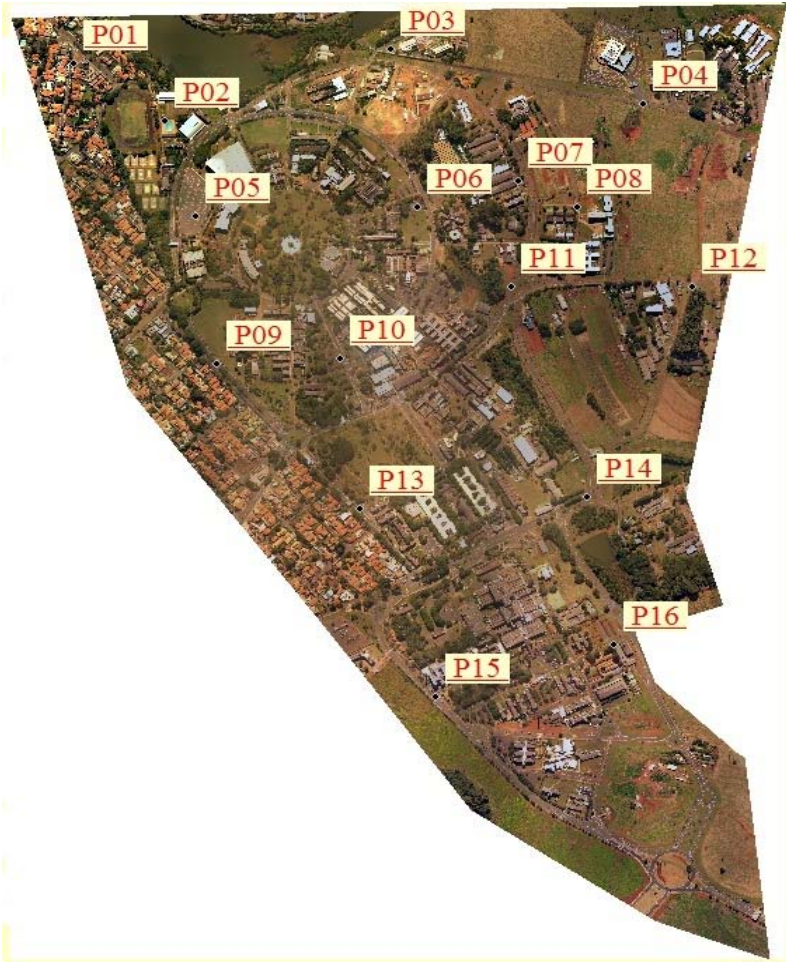

Fig. 3 Distribution of control points in the studied area. mark M03 from the Unicamp registration reference network. The data were post-processed using Topcon Tools software to achieve precision sufficient for a 1:1,000 scale according to a CES (Cartographic Accuracy Standard) grade of "A". The positions for these points were verified using the Global Mapper program (version 11), which was loaded with the orthophotos, field-surveyed points and digital ground model from the laser system. Coordinates for the points of interest were extracted via a visual analysis of the orthophotos and compared to those of the GNSS field survey shown in Tables 2 and 3.

When generating cartographic products (orthophotos and digital ground models), DATUM SAD69 was used as a reference. Therefore, the field data were converted to this projection system using the TCGeo software (version 6.1), which was developed by the Brazilian Institute of Geography and Statistics (Instituto Brasileiro de Geografia e Estatística

Table 2 Comparison of field data, orthophotos and the digital ground model, Part 1.

\begin{tabular}{llllll}
\hline Point & $h$-Ellipsoidal & $E(\mathrm{~m})$ & $N(\mathrm{~m})$ & Geoidal undulation $N$ & $H=h-N$ \\
\hline P01 & 588.70 & $287,053.91$ & $7,475,786.38$ & 2.36 & 586.34 \\
P02 & 589.23 & $287,293.26$ & $7,475,625.66$ & 2.37 & 586.86 \\
P03 & 593.13 & $287,877.85$ & $7,475,826.72$ & 2.39 & 590.74 \\
P04 & 628.98 & $288,535.60$ & $7,475,671.60$ & 2.41 & 626.57 \\
P05 & 595.79 & $287,374.16$ & $7,475,358.24$ & 2.38 & 593.41 \\
P06 & 588.91 & $287,949.71$ & $7,475,384.48$ & 2.40 & 586.51 \\
P07 & 605.62 & $288,211.02$ & $7,475,459.40$ & 2.40 & 603.22 \\
P08 & 620.99 & $288,361.40$ & $7,475,384.09$ & 2.41 & 618.58 \\
P09 & 601.88 & $287,428.38$ & $7,474,948.49$ & 2.39 & 599.49 \\
P10 & 605.16 & $287,748.98$ & $7,474,962.33$ & 2.40 & 602.76 \\
P11 & 599.63 & $288,191.38$ & $7,475,161.91$ & 2.41 & 597.22 \\
P12 & 645.29 & $288,662.72$ & $7,475,164.50$ & 2.42 & 642.87 \\
P13 & 612.95 & $287,796.99$ & $7,474,543.61$ & 2.41 & 610.54 \\
P14 & 604.91 & $288,388.51$ & $7,474,577.60$ & 2.42 & 602.49 \\
P15 & 624.46 & $287,997.03$ & $7,474,020.87$ & 2.42 & 622.04 \\
P16 & 627.06 & $288,458.27$ & $7,474,164.56$ & 2.43 & 624.63 \\
\hline
\end{tabular}


Table 3 Comparison of the field data, orthophotos and digital ground model, Part 2.

\begin{tabular}{lllllll}
\hline E/Orthophoto & $N /$ Orthophoto & $H /$ dem & $\# E$ & $\# N$ & \# Planimetry & $\# H$ \\
\hline $287,054.15$ & $7,475,786.99$ & 586.09 & -0.25 & -0.61 & 0.66 & 0.25 \\
$287,293.00$ & $7,475,625.71$ & 586.68 & 0.25 & -0.05 & 0.26 & 0.18 \\
$287,877.72$ & $7,475,827.02$ & 590.56 & 0.13 & -0.31 & 0.33 & 0.18 \\
$288,535.66$ & $7,475,671.86$ & 625.93 & -0.06 & -0.27 & 0.27 & 0.65 \\
$287,374.34$ & $7,475,357.81$ & 593.16 & -0.17 & 0.43 & 0.46 & 0.25 \\
$287,949.60$ & $7,475,384.15$ & 586.23 & 0.12 & 0.33 & 0.35 & 0.28 \\
$288,210.98$ & $7,475,459.71$ & 603.11 & 0.03 & -0.32 & 0.32 & 0.11 \\
$288,361.95$ & $7,475,384.14$ & 618.00 & -0.56 & -0.05 & 0.56 & 0.58 \\
$287,427.95$ & $7,474,947.87$ & 599.20 & 0.43 & 0.62 & 0.75 & 0.29 \\
$287,748.37$ & $7,474,961.67$ & 602.50 & 0.61 & 0.66 & 0.90 & 0.26 \\
$288,191.89$ & $7,475,161.64$ & 597.10 & -0.51 & 0.28 & 0.58 & 0.12 \\
$288,662.69$ & $7,475,163.72$ & 642.08 & 0.02 & 0.79 & 0.79 & 0.79 \\
$287,797.28$ & $7,474,543.50$ & 610.20 & -0.29 & 0.11 & 0.31 & 0.34 \\
$288,388.57$ & $7,474,577.71$ & 602.14 & -0.06 & -0.11 & 0.13 & 0.35 \\
$287,997.52$ & $7,474,020.79$ & 621.79 & -0.49 & 0.08 & 0.50 & 0.25 \\
$288,458.75$ & $7,474,164.42$ & 624.51 & -0.48 & 0.14 & & 0.12 \\
\hline
\end{tabular}

-IBGE). The orthometric altitude was obtained using the Mapgeo 2004 software (version 3.0), also developed by IBGE.

\section{Conclusions}

Consideration of Decree 89,817 of June 20, 1984 [8], which regulated Legislative Decree No. 243/67 and established the quality control for cartographic products and the CES, indicates that the products generated met the CES class " $A$ " requirements for a $1: 2,000$ scale because $90 \%$ of the well-defined points in the cartographic record that was tested on the ground showed an error below the CES-Planimetry limit of $0.5 \mathrm{~mm}$ and a standard deviation below 0.3 $\mathrm{mm}$ on the map scale. These values represent $1.0 \mathrm{~m}$ and $0.6 \mathrm{~m}$ on the ground, respectively.

The altimetry data obtained via the laser scanner system also met the classification prerequisites for the $1: 2,000$ scale because $90 \%$ of its points were below $50 \%$ of the equidistance contour lines, $2 \mathrm{~m}$ in this case, and $1 / 3$ were considered within the standard deviation.

Thus, the process used herein achieved results compatible with cartographic products on the 1:2,000 scale (orthophotos) and represented a reduction in the cost and time of mapping.
For this study in question, only the positioning of the final generated mosaic was considered. Cases with parallax between the photos were not analyzed and should be the subject of further study.

\section{References}

[1] D.C. Costa, Guidelines for elaborating and using cartographic databases in municipal planning: Urban, rural and transportation, Dr. Thesis, Polytechnic School of the University of São Paulo, São Paulo, 2001.

[2] J.D. Anderson, Aircraft Performance and Design, McGraw-Hill, Boston, 1999.

[3] J. Skaloud, Optimizing georeferencing of airbone survey systems by INS/DGPS, Ph.D. Thesis, University of Calgary, 1999.

[4] K.P. Schwarz, M.A. Chapman, M.E. Cannon, P. Gong, An integrated INS/GPS: Approach to the georeferencing of remotely sensed data, Photogrammetric Engineering and Remote Sensing 59 (11) (1993) 1667-1674.

[5] J. Brito, L. Coelho, Digital Photogrammetry, 1st ed., Military Institute of Engineering, Rio de Janeiro, 2002.

[6] M. Cramer, Integrated GPS/inertial and digital aerial triangulation-Recent test results, in: Photogrammetric Week 2003, Herbert Wichmann Verlag, Heidelberg, 2013, pp. 161-172.

[7] V. Casella, M. Franzini, Definition of a methodology for local reduction of parallaxes in directly oriented images, in: Proceedings of International Society for Photogrammetry and Remote Sensing Workshop, 
Castelldefels, Spain, 2003.

[8] Decree No. 89817, Details: The Regulatory Instructions for the Technical Standards of the National Cartography, Official Gazette of the Federal Republic of Brazil, 1984. 